\title{
Hedges enhance memory but inhibit retelling
}

\author{
Kris Liu • Jean E. Fox Tree
}

Published online: 14 June 2012

(C) Psychonomic Society, Inc. 2012

\begin{abstract}
We examined the effects of hedges and the discourse marker like on how people recalled specific details about precise quantities in spontaneous speech. We found that listeners treated hedged information differently from like-marked information, although both are thought to be indicators of uncertainty or vagueness. In addition, hedges had different effects depending on whether speakers were (1) retelling conversations to another person or (2) answering questions about material they had heard. When retelling to another person, listeners were more likely to report information that was either unmarked or marked with a like than hedged information (Experiment 1). Yet when answering questions by themselves, hedges enhanced memory for details, in comparison with likes (Experiment 2). Hedges appear to provide pragmatic cues about what information is reliable enough to repeat in a conversational context. But although hedged information may be left out, it is not forgotten.
\end{abstract}

Keywords Hedges - Likes · Discourse markers · Spontaneous speech $\cdot$ Memory

\section{Hedges enhance memory but inhibit retelling}

Typically, the information people carry away from a conversation contains the gist and perhaps some funny or

\section{K. Liu $(\varangle) \cdot$ J. E. Fox Tree}

Psychology Department, University of California,

Social Sciences II room 277,

Santa Cruz, CA 95064, USA

e-mail: kyliu@ucsc.edu

J. E. Fox Tree

e-mail: foxtree@ucsc.edu particularly memorable verbatim (or close to verbatim) tidbits (Bartlett, 1932; Keenan, MacWhinney, \& Mayhew, 1977). In one study, people retold only half the details from a story they read (Dudukovic, Marsh, \& Tversky, 2004). The details chosen for retelling were similar across those who retold for accuracy and those who retold for entertainment. We propose that in spoken, spontaneous stories, hedges (such as maybe, I think, and I dunno) influence which small, specific details (such as information about precise quantity of objects, time, or space) are remembered and retold. We provide evidence that hedges mark information as unreliable, while at the same time enhancing memory for that information. We also provide evidence that the discourse marker like is not a hedge, despite the popular notion that its use connotes vagueness (Jucker, Smith, \& Lüdge, 2003).

While prevalent, hedges and likes are not considered desirable additions to spoken utterances. Like has both negative connotations and negative effects. It was thought to hinder communication by about a third of respondents in one study, and about $80 \%$ reported avoiding its use at times (Fox Tree, 2007). It can make speakers appear less educated (Daily-O'Cain, 2000) and can reduce the likelihood of getting a job when overused (Russell, Perkins, \& Grinnell, 2008). Hedges are often classified as powerless language and, along with other powerless elements, such as hesitations and tag questions, have been shown to decrease ratings of a speaker's perceived confidence, competence, and attractiveness (Blankenship \& Holtgraves, 2005). While it is possible that speakers use hedges to buy time when they need to retrieve information, this cannot always be the case: Many hedges are appended after a quantity.

Likes and hedges can, however, have positive effects. Likes can make people appear more friendly and attractive (Daily-O'Cain, 2000) and can improve the exchange of 
information in an interview setting (Fuller, 2003). Hedges can also provide necessary information in a conversation. For example, probably and I guess are thought to indicate a speaker's lack of certainty about information presented or to soften a speaker's commitment to an utterance (Jucker et al., 2003).

One approach to hedges and likes is that they detract from information they mark, causing listeners to consider hedged and like-marked information as less reliable and, therefore, less worthy of noting and remembering. The alternative is that hedges and likes provide important information, their modifying presence perhaps focusing attention on the information they mark (see Underhill, 1988, for a discussion of like), making that material more worthy of note and recall. Although a focusing-attention function has not been demonstrated experimentally for hedges and likes, other spoken signals, such as oh and $u h$, have been shown to affect listeners' ability to access information (Fox Tree, 1995; Fox Tree \& Schrock, 1999).

Most previous work on hedging has centered on the impact of hedges (and other markers of powerlessness or uncertainty) on the persuasiveness of an argument, rather than the memorability of the information presented. For example, written arguments using powerless language were judged more negatively than those without (Hosman, Huebner, \& Siltanen, 2002). Also, hedged speech negatively influenced the persuasiveness of an argument (Blankenship \& Holtgraves, 2005). The one test of the effect of hedges on memory compared recall of persuasive arguments. In contrast to the intuitive idea that people would feel more confident about the truth and reliability of unhedged information over hedged information and, therefore, pay more attention to unhedged information, readers had more thoughts on hedged statements and tended to remember the information at a higher rate (Hosman \& Siltanen, 2006). No researchers have tested the role of hedges in the memory of everyday conversations.

There are several different categories of hedges, such as vague quantifying expressions (some, many), adverbs of frequency (sometimes, usually), adverbs of likelihood (probably, maybe), approximators (about, around), propositional attitudes (I think, I dunno), and phrases such as or something [like that]. The low sample size for each category in our spontaneously produced material precluded testing subsets of hedges. But one sometimessubset was testable: the use of like.

We say sometimes-subset because, although likes are often lumped in with hedges, there is doubt about whether or not likes are hedges in the sense of estimation or lack of certainty (see the discussions in Fox Tree, 2006, 2007). An alternative possibility is that likes highlight that what is said is only a partial reflection of what the speaker intends (Anderson, 1998; Schourup, 1985; Siegel, 2002), a discourse marker used to indicate an upcoming loose use of language (Fox Tree, 2006). Unlike other items called hedges, like cannot be equated to approximately (Anderson, 1998; Fox Tree, 2006; Fuller, 2003; Siegel, 2002; Underhill, 1988).

Previous work on like has also not tested how like might influence the memorability of subsequent information in a conversational setting. Like hedges, the use of like can also be considered a manner of expressing vagueness (Jucker et al., 2003). It is also commonly associated with the speech of immature and unintelligent (and presumably less powerful) people (for a review, see Fox Tree, 2006). The interpretation of information marked with like by both listeners and speakers might be predicted to resemble the interpretation of information marked with more conventional hedges: In contexts where specific numbers are used, the use of a hedge or a like might mark that particular piece of information as less reliable or less accurate, in comparison with unmarked information. But because likes are argued to not be hedge equivalents, they may have different effects from hedges.

Specifically, we predict that because hedges mark estimation or lack of certainty, hedged information will be interpreted as less important elements of a story line and, consequently, be less likely to be included in a retelling of that story. However, the presence of the hedges might, at the same time, cause listeners to think more deeply about hedged-marked information, therefore increasing memorability (Hosman \& Siltanen, 2006). In contrast, because likes mark a loose use of language at moments where that looseness is necessary (cf. Fox Tree, 2006), likes may not decrease the likelihood of including like-marked information in a retelling. At the same time, likes may also cause listeners to think more deeply about like-marked information, to determine what the speaker's fuller intent is, increasing memorability of like-marked concepts.

To test the effect of hedges (including the potential hedge like) on memory, we compared (1) the rate of repetition and (2) the accuracy of repetition of multiple tellings of specific numeric information (quantities) that occurred in spontaneously produced autobiographical stories (Experiment 1) and the effect of hedges and likes on the recall of story details (Experiment 2). Hedged quantities were used because they offer a more rigorous measurement of whether the quantity was included in the retelling and how close it was to the original.

If speakers use hedges to indicate information that is unreliable, information marked with a hedge should be less likely than unmarked information to be retold in subsequent tellings of the same story. Furthermore, if like is a hedge (and is thus unreliable), information that is marked with a like will also be dropped in subsequent tellings.

There is reason to think that speakers will treat hedges and likes in retelling their own stories differently from 
retelling others' stories. Speakers retelling their own stories can be seen as performing primarily a production task. Their hedges and likes might be expected to occur at similar rates, possibly in similar locations. Speakers retelling others' stories can be seen as performing comprehension and production tasks. They might encode marked information poorly and, therefore, be less likely to remember and retell that information than are the stories' creators. However, there is also reason to expect original-story-tellers and others'-storytellers to treat marked information similarly. When retelling stories, speakers recycled the position of $22 \%$ of their own likes and $28 \%$ of others' likes (Fox Tree, 2006). Either relationship can be observed without damaging our principal comparison, which is the frequency of reproduction of hedged, like-marked, and unmarked information.

\section{Experiment 1}

Method

Participants Twenty-two University of California (UC), Santa Cruz undergraduates participated in exchange for course credit.

Procedure Each session involved 2 naïve participants (assigned as A and B) and 2 research assistants (RA1 and RA2). There were four audio-recorded parts in order to produce six tellings of two stories (one story belonging to $\mathrm{A}$ as speaker with $\mathrm{B}$ as listener, one story belonging to $\mathrm{B}$ as speaker with $\mathrm{A}$ as listener). Each story was told twice by its original speaker (originalspeaker telling and speaker retelling) and repeated once by the listener (listener retelling).

In part 1, A (acting as speaker) told an autobiographical story for about 12 min about "a large purchase made in the last year" to B (acting as listener). In part 2, A stayed in the same room, and B was taken to another room. While A retold his or her own story to RA1, B recalled as much of A's story as possible to RA2.

Parts 3 and 4 mirrored parts 1 and 2. In part 3, B and A were reunited, but this time B (as speaker) told an autobiographical story about "a memorable conversation" to A (as listener). In part $4, \mathrm{~A}$ and $\mathrm{B}$ were again separated into different rooms with the RAs they had already spoken with: $\mathrm{B}$ repeated the original-speaker telling story to RA2, while A recalled the story to RA1.

Coding After transcription, phrases that contained quantities (e.g., "I got home at 5 a.m.") were identified in the original-speaker telling condition and speaker retell and listener retell conditions. Retellings were examined to determine whether quantities from the original telling were included in the subsequent tellings (e.g., "She didn't get home until 5 a.m." or "She didn't get home until it was super late, like the sun was rising"). A vaguer retelling was acceptable, but only if it exclusively referred to a clearly identifiable instance from the first story. The quantityrelated information in the original-speaker telling was coded for the presence of a like, a hedge, both, or neither. Retellings were not assessed for the presence of likes, hedges, both, or neither. The amount of similar quantities across the retellings was counted, grouped by how they were presented in the original-speaker telling - with a like, a hedge, both, or neither. Categories of quantities and hedges assessed are given in the Appendix.

Results

Overwhelmingly, participants repeated information similarly across all tellings, regardless of whether they were in the speaker or listener role. Of all the quantities that were repeated (216 for speakers, 185 for listeners), only 13 were retold in a way that was different from their first telling (i.e., the retelling's quantity did not match the quantity used in the original telling): 4 by speakers and 9 by listeners. Although the use of hedges and likes in retellings was not assessed, we observed that all of the quantities that were retold differently from the original contained a hedge in the original telling. Table 1 presents the frequencies of each coding category.

Two logistic regression analyses were conducted to predict the inclusion of a quantity in (1) speaker-retold stories and (2) listener-retold stories, with (a) presence of like in the original (yes, no), (b) presence of a hedge in the original (yes, no), (c) interaction between presence of like and presence of hedge, (d) story type (large purchase, memorable conversation), and (e) conversational pair as predictors. Tests of the full models against constant-only models were statistically significant, indicating that the predictors as a set reliably distinguished between inclusion and exclusion of a quantity, $\chi 2(16)=41.87, p<.001$, for speaker-retold stories and $\chi^{2}(16)=61.03, p<.001$, for listener-retold stories. Prediction success overall was $68.7 \%$ (49.2\% for exclusion, $80.7 \%$ for inclusion) for speaker retellings and $67.5 \%$ (63.2\% for exclusion and $71.5 \%$ for inclusion) for listener retellings.

Table 1 Frequencies of quantity-related statements in the original telling across all speakers by category (with percentage of total in parentheses)

\begin{tabular}{llll}
\hline Hedge & Like & Hedge + Like & None \\
\hline 40 & 93 & 58 & 152 \\
$(11.7 \%)$ & $(27.1 \%)$ & $(16.9 \%)$ & $(44.3 \%)$ \\
\hline
\end{tabular}


For speaker retellings, the Wald criterion demonstrated that presence of a like did not make a significant contribution to prediction, Wald $=0.01, p=.906$, but that presence of a hedge did, Wald $=3.77, p=.052$. There was no interaction between like and hedge, Wald $=0.11, p=.741$. The regression indicated that when a hedge was present in the original telling, tellers were almost half as likely to include the quantity in retelling their own story (.47 times). Story type did not make a significant contribution to prediction, Wald $=2.24, p=.135$. Conversational pairs did make a significant contribution to prediction, Wald $=26.02$, $p=.011$. We interpret this as indicating that conversational pairs varied in how conscientious they were about reporting details.

Similarly, for listener retellings, the Wald criterion demonstrated that presence of a like did not make a significant contribution to prediction, Wald $=3.17, p=.075$, but that presence of a hedge did, Wald $=14.19, p<.001$. There was no interaction between like and hedge, Wald $=0.04, p=.852$. The regression indicated that when a hedge was present in the original telling, tellers were about a fifth as likely to include the quantity when retelling someone else's story (.22 times). As with speaker retellings, story type did not make a significant contribution to prediction, Wald $=1.29, p=.255$. Also as with the speaker retellings, conversational pairs made a significant contribution to prediction, Wald $=22.81, p=.029$. As before, we interpret this as indicating that conversational pairs varied in how conscientious they were about reporting details.

To graphically display the results, the percentage of quantities retold in each marking category was calculated. Figure 1 shows the mean percentage of quantities retold by speaker and listener, broken down by whether it was hedged, like-marked, both, or unmarked.

\section{Discussion}

Unsurprisingly, participants included more quantities in retelling their own stories than in retelling others' stories. This could be because they simply remembered their own quantities better. Also unsurprisingly, some conversational pairs reported more quantities than did others.

Less predictably, hedge-alone and hedge + like quantities were less likely to be retold than unmarked quantities or like-alone quantities. This was true regardless of whether speakers were retelling their own story or another's story. This suggests three things. First, speakers use hedges to mark information as less worthy of repetition, and listeners interpret hedged information accordingly (i.e., they fail to repeat it). Second, while like is often considered a hedge, like does not have the same impact on retelling of details from a dialogue as a hedge does. Third, the reason like may be so often mistaken for a hedge is because like can co-occur with hedges. In this corpus, the co-occurring hedge + like marking on quantities is slightly more prevalent than hedgealone.

Hedges may be reflective of the speaker's confidence or may accompany details that the speaker judges as less interesting or informative: The speaker may have thought those details were worthy of telling but, after the initial telling, may have decided that they were unworthy of repetition. For listeners, hedges might operate as an online cue during comprehension that directly influences memory processes, possibly cuing them to ignore the information that is
Fig. 1 Mean percentage of quantities retold by speaker and listener in Experiment 1

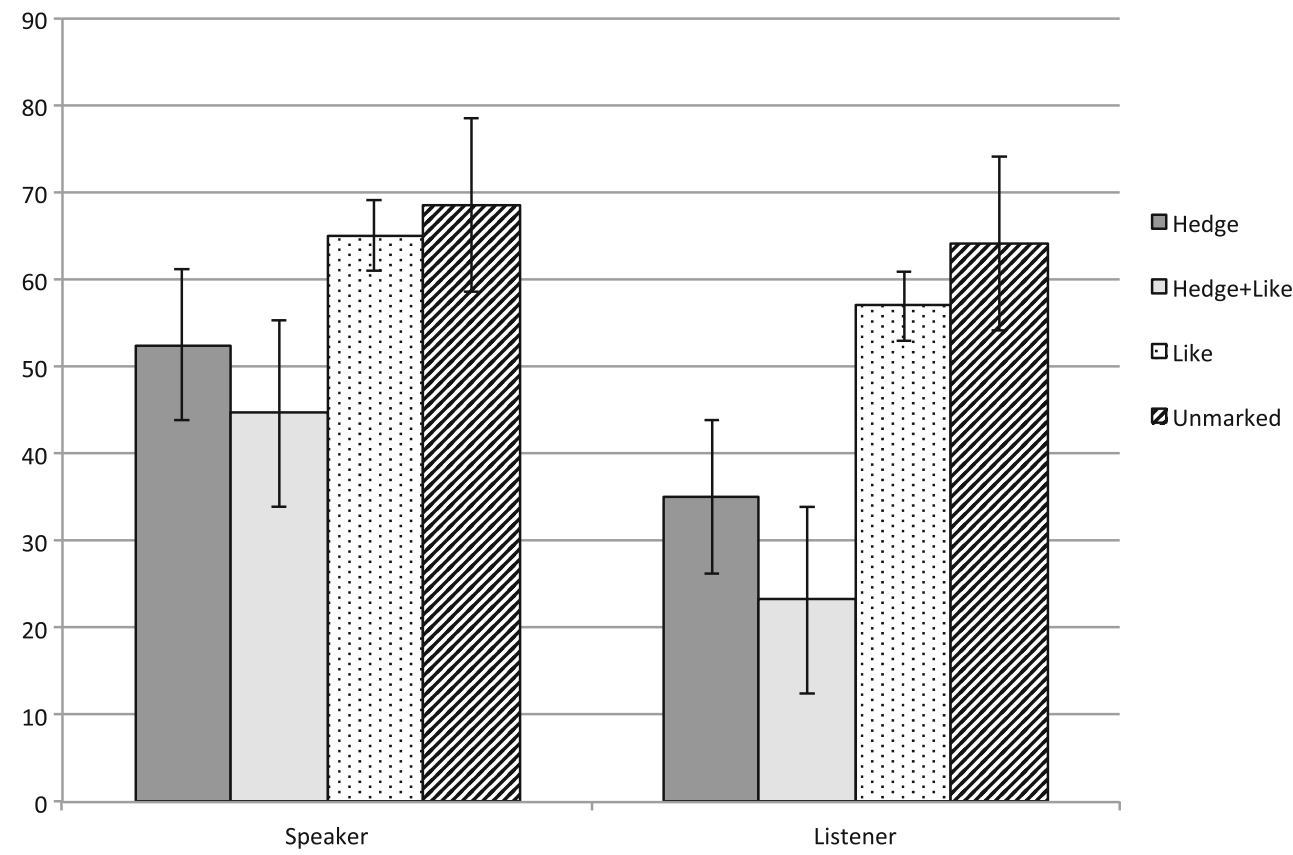


presumably being marked as uncertain, inaccurate, or perhaps as less relevant than unmarked or like-marked information. Alternatively, hedges might operate as a pragmatic or procedural cue that induces listeners to not repeat to an interlocutor details that have been marked as uncertain, although the listener has retained the hedged information.

In Experiment 2, we tested whether hedges negatively affected memory encoding or retrieval of conversational information. If hedges negatively affect memory, listeners should have trouble remembering hedged-marked information, in comparison with the same information presented without hedges. Alternatively, hedges may not negatively affect encoding or retrieval. This pattern would suggest that hedges operate on a pragmatic level, flagging information as less relevant to include in a retelling, but not preventing the information from being remembered.

In Experiment 2, we also tested whether likes negatively affected memory for information in conversations. Because like-alone-marked information was retold just as often as unmarked information, we expected like-alone-marked information to be accurately remembered and identified at least as easily as the same information presented without likes.

\section{Experiment 2}

\section{Method}

Participants Eighty-one UC Santa Cruz undergraduates participated in exchange for course credit.

Procedure An 8-min audio excerpt of an original-speaker's telling from the Experiment 1 corpus was selected, and 18 target quantities were identified: 6 hedge-marked, 6 likemarked, and 6 unmarked quantities that were used as fillers. Unlike in Experiment 1, the combined hedge + like marking was not tested. There were several instances where a quantity was marked with both, but these were edited so that each quantity was marked with only a hedge, only a like, or neither. The excerpt contained four additional unedited, nontarget quantities.

Two versions of the dialogue were created. In version A, the likes from the like-marked target items were removed, but the hedge-marked items were left as is. In version B, the hedges from the hedge-marked items were removed, but the like-marked items were left as is. The unmarked items were unchanged across conditions.

Participants were randomly assigned to version A or B. They listened to the dialogue and completed an 18-item quiz testing memory accuracy for the quantities. For instance, a participant might be asked, "How much did the speaker in the story pay for the jacket?" and the participant would have to choose from one of four prices that were in the same range as the actual price. The order of the questions was randomized for each participant.

To ensure that the audio sounded natural, edits were made only to quantities that could be made to sound as if they had not included a hedge or like. To test the detectability of editing, 18 other participants listened to the stimuli. They were explicitly told that the audio had been edited and that their task was to identify every location that they suspected of having edits. Many different locations were identified as having been edited, yet the vast majority of suspected locations contained absolutely no editing. Of the total 108 points ( 6 per participant) where hedges or likes had been spliced out, only 5 hedge-splice points and no like splice points were correctly identified. This low level of detectability is common in our laboratory (Fox Tree, 1995, 2001; Fox Tree \& Schrock, 1999) and suggests that any results found are not due to editing effects. Finally, participants in Experiment 2 were informed postexperiment that some specific elements of the dialogue had been excised. When asked to guess what had been removed, none identified the experimental manipulation, for either hedges or likes.

\section{Results}

A $2 \times 2$ mixed design ANOVA was run to examine the interaction between the items originally hedged or likemarked and story version. There was a significant interaction (with no main effect of marking), $F(1,79)=4.13$, $p=.045$.

Planned comparisons were run to compare accuracy rate for hedged items and for like-marked items between version A and version B. Participants were more likely to accurately remember information that was originally hedged when the hedge was intact than when it was excised. When the information was hedged, participants got an average score of $75 \%$. When those hedges were removed, the score dropped to $64 \%, t(79)=2.36, p=.021$.

Accuracy for like-marked information, however, was the same rate regardless of like presence: $77 \%$ with like and $73 \%$ without, $t(79)=-.31, p=.757$.

\section{Discussion}

Although Experiment 1 suggested that hedge-marked information may have been overlooked by listeners when they were listening to the stories, Experiment 2 demonstrated that hedged information was more accurately remembered than the same information presented without a hedge. Like had no effect on information retrieval. 


\section{General discussion}

The two experiments in this study appear to contradict one another on the influence of hedges on memory for conversation. Yet they are not incompatible. We propose that hedges provide pragmatic cues about what information is reliable enough to repeat to somebody else in a conversational context, but they do not prevent people from remembering that information.

Following Hosman and Siltanen's (2006) finding that people think more about powerless language, we propose that hedges make people think more about hedged information. Increased consideration may act as a form of rehearsal, which, in turn, enhances memory. Yet hedges also mark information and speakers as less reliable (Hosman \& Wright, 1987), which would decrease motivation for listeners to repeat such information in a subsequent conversation.

Exactly how rehearsal, source credibility, and motivation affect the retelling of narratives is largely unknown. In one study, adding details and contextual information did not induce people to retell an urban legend (by increasing credibility and, therefore, the likelihood of retelling), although people did include the extra information if they chose to retell a legend that had been presented with it (demonstrating that the information was encoded; Fox Tree \& Weldon, 2007). In another study, a claim repeatedly marked as false (which should, therefore, reduce credibility) was recalled as truthful 3 days later, a result of mistakenly taking familiarity as truthfulness (revealing a disadvantage of rehearsal; Skurnik, Yoon, Park, \& Schwarz, 2005). A third study by Nabi, Moyer-Gusé, and Byrne (2007) on humor may offer the most useful analog for the influence of hedges. Humorous narratives were more vulnerable to having their persuasive content discounted, which suggests a possible negative effect on message recall. However, the more humorous the message, the more deeply it was processed, which, in turn, may have a positive effect on recall. The present study suggests that hedges increase processing (leading to more accurate recall), while at the same time encouraging discounting (leading to less frequent inclusion in a retelling), but as with other studies, it is not exactly clear how this comes about.

Although precision can be less efficient (and costlier) than vagueness (Gibbs \& Bryant, 2008), hedging may still have a cost. Appending or something immediately after naming the amount of money one has spent on an expensive pair of sneakers can be interpreted, at first glance, as impractical and inefficient. It does not save the speaker from seeming frivolous, because it still reveals the ballpark price range, and it may induce the listener to believe that the speaker is a source of inaccurate information (cf. Hosman $\&$ Siltanen, 2006). Including a hedge in this situation may seem strange, and this strangeness may contribute to the memorability of hedge-marked information, similar to how a bizarre sentence enhances memory for ordinary nouns within the sentence (Macklin \& McDaniel, 2005). That is, an unnecessary hedge may lead a listener to pay more attention to information, and if a listener is led to give more consideration as to why a hedge was used, this closer examination may render the information more easily accessible for future recall.

These two experiments cannot distinguish between whether hedges strengthen memory because listeners more closely evaluate a speaker's reliability (cf. Hosman \& Wright, 1987), because hedges catch a listener's attention through their potentially unnecessary presence, or because people are more likely to listen for cues that tell them when a speaker wants them to pay attention rather than when not to pay attention (cf. Fox Tree, 2001; Fox Tree \& Schrock, 1999). Regardless, hedges seem to encourage the listener to think about what the speaker has said or is saying, which increases the chance of later recall.

The present study also provides evidence against the notion that like functions as a hedge. Like-marked information was retold and recalled differently from hedged information. Although we found evidence that hedges focus attention, we did not find the same for like. A priori, we predicted that likes would improve memory, since like may encourage listeners to think about the looseness-implications of like-marked information. When a speaker says "it's like a $2 \mathrm{~h}$ drive," a listener may try to understand exactly what is being loosely expressed, such as " $\mathrm{a} 2 \mathrm{~h}$ drive is too long for something unimportant," or the opposite, "that's a short drive for something important." However, to be properly understood, like-marked information may require more familiarity between interlocutors than the participants in the present experiments had. For example, for "it's like a $2 \mathrm{~h}$ drive," the addressee may need to know what the speaker's thoughts are about 2-h drives. Future studies may uncover an effect of like on conversational memory by exploring communication between close friends.

Author Note This research was supported by faculty research funds granted by the University of California, Santa Cruz. We thank our many research assistants who aided in data collection and coding, with a special thanks to Brian Dillon, Dara Pastor, and Aileen Giebitz. We would also like to also thank our anonymous reviewers for valuable comments on an earlier draft of the manuscript.

\section{Appendix}

\section{Quantities}

a. Hours; e.g., "It was like a $2 \mathrm{~h}$ drive" and "I'd been there since seven [a.m.]."

b. Months and weekdays; e.g., "In January" and "It was a Thursday." 
c. Times of year and day; e.g., "I went [camping] last summer" and "We got in the car early in the morning."

d. Years; e.g., "The 1984 Olympics."

e. Academic years; e.g., "In my sophomore year" or "When I was, I think, in fourth grade."

f. Cost; e.g., "Charged me like 200, 300 bucks."

g. Spatial measurement; e.g., "40 foot up waterfall” and "The trail ends like halfway there."

h. Precise amounts; e.g., "Eight dads and their daughters."

i. Imprecise amounts; e.g., "By the end of the week" and "A couple of days."

j. Ordinal indicators; e.g., "The week before [the concert]" and "The first time I ever went horseback riding."

k. "Double" and "triple"; e.g., "Then I moved into a triple [dorm room]."

\section{Hedges}

a. Vague quantifying expressions; e.g., some, many.

b. Adverbs of frequency; e.g., sometimes, usually.

c. Adverbs of likelihood; e.g., probably, maybe.

d. Approximators; e.g., about, around.

e. Propositional attitudes; e.g., I think, I guess, I don't know.

f. "Or something [like that]."

\section{References}

Anderson, G. (1998). The pragmatic marker like from a relevance-theoretic perspective. In A. H. Jucker \& Y. Ziv (Eds.), Discourse markers: Description and theory (pp. 147-170). Amsterdam: Benjamins.

Bartlett, F. C. (1932). Remembering: A study in experimental and social psychology. Cambridge: Cambridge University Press.

Blankenship, K., \& Holtgraves, T. (2005). The role of different markers of linguistic powerlessness in persuasion. Journal of Language and Social Psychology, 24, 3-24.

Daily-O'Cain, J. (2000). The sociolinguistic distribution of and attitudes toward focuser like and quotative like. Journal of Sociolinguistics, 4, 60-80.

Dudukovic, N. M., Marsh, E. J., \& Tversky, B. (2004). Telling a story or telling it straight: The effects of entertaining versus accurate retellings on memory. Applied Cognitive Psychology, 18, 125-143.
Fox Tree, J. E. (1995). The effects of false starts and reptitions on the processing of subsequent words in spontaneous speech. Journal of Memory and Language, 34, 709-738.

Fox Tree, J. E. (2001). Listeners' uses of um and uh in speech comprehension. Memory \& Cognition, 29, 320-326.

Fox Tree, J. E. (2006). Placing like in telling stories. Discourse Studies, $8,723-743$.

Fox Tree, J. E. (2007). Folk notions of um and uh, you know and like. Text \& Talk, 27, 297-314.

Fox Tree, J. E., \& Schrock, J. C. (1999). Discourse markers in spontaneous speech: Oh what a difference an oh makes. Journal of Memory and Language, 40, 280-295.

Fox Tree, J. E., \& Weldon, M. S. (2007). Retelling urban legends. The American Journal of Psychology, 120, 459-476.

Fuller, J. M. (2003). Use of the discourse marker like in interviews. Journal of Sociolinguistics, 7, 365-377.

Gibbs, R. W., \& Bryant, G. (2008). Striving for optimal relevance when answering questions. Cognition, 1, 345-369.

Hosman, L. A., Huebner, T. M., \& Siltanen, S. A. (2002). The impact of power-of-speech style, argument strength, and need for cognition on impression formation, cognitive responses, and persuasion. Journal of Language and Social Psychology, 21, 361-379.

Hosman, L. A., \& Siltanen, S. A. (2006). Powerful and powerless language forms: Their consequences for impression formation, attributions of control of self and control of others, cognitive responses, and message memory. Journal of Language and Social Psychology, 25, 33-46.

Hosman, L. A., \& Wright, J. W. (1987). The effects of hedges and hesitations on impression formation in a simulated courtroom context. Western Journal of Speech Communication, 51, 173188.

Jucker, A. H., Smith, S. W., \& Lüdge, T. (2003). Interactive aspects of vagueness in conversation. Journal of Pragmatics, 35, 17371769.

Keenan, J. M., MacWhinney, B., \& Mayhew, D. (1977). Pragmatics in memory: A study of natural conversation. Journal of Verbal Learning and Verbal Behavior, 16, 549-560.

Macklin, C., \& McDaniel, M. (2005). The bizarreness effect: Dissociation between item and source memory. Memory, 13, 682-689.

Nabi, R., Moyer-Gusé, E., \& Byrne, S. (2007). All joking aside: A serious investigation into the persuasive effects of funny social issue messages. Communication Monographs, 74, 29-54.

Russell, B., Perkins, J., \& Grinnell, H. (2008). Interviewees' overuse of the word "like" and hesitations: Effects in simulated hiring decisions. Psychological Reports, 102, 111-118.

Schourup, L. (1985). Common discourse particles in English conversation. New York: Garland.

Siegel, M. (2002). Like: The discourse particle and semantics. Journal of Semantics, 19, 35-71.

Skurnik, I., Yoon, C., Park, D., \& Schwarz, N. (2005). How warnings about false claims become recommended. Journal of Consumer Researcher, 31, 713-724.

Underhill, R. (1988). Like is, like, focus. American Speech, 63, 234 246. 